\title{
Stakeholder engagement in the management of indoor radon exposures
}

\author{
C. Turcanu ${ }^{1, *}$, C. Schieber ${ }^{2}$, T. Schneider ${ }^{2}$, C. Fallon ${ }^{3}$, R. Geysmans ${ }^{1}$, T. Perko ${ }^{1}$, M-C. Cantone ${ }^{4}$, S. \\ Economides $^{5}$, F. Barazza ${ }^{6}$, S. Charron ${ }^{7}$, R. Gschwind ${ }^{8}$, S. Lafage ${ }^{2}$, N. Zeleznik ${ }^{9}$ and C. Pölzl-Viol ${ }^{10}$ \\ ${ }^{1}$ SCK-CEN-Belgian Nuclear Research Centre, Boeretang 200, 2400 Mol, Belgium. \\ 2 CEPN-Nuclear Protection Evaluation Centre, Fontenay-aux-Roses, France. \\ ${ }^{3}$ University of Liège, Faculty of Law, Place des Orateurs 3, 4000 Liège, Belgium. \\ ${ }^{4}$ University of Milan, Via Pascal 36, 20133 Milan, Italy. \\ ${ }^{5}$ EEAE-Greek Atomic Energy Commission, Agia Paraskevi, Athens, Greece. \\ ${ }^{6}$ Federal Office of Public Health, Schwarzenburgstrasse 157, 3003 Bern, Switzerland. \\ ${ }^{7}$ IRSN-Institut de Radioprotection et de SÛreté Nucléaire, Fontenay-aux-Roses, France. \\ ${ }^{8}$ Université Franche Comté, Laboratoire Chrono-environnement, Montbéliard, France. \\ 9 EIMV, Milan Vidmar Electric Power Research Institute, Hajdrihova 2, Ljubljana, Slovenia. \\ ${ }^{10}$ BfS - Federal Office for Radiation Protection, Salzgitter, Germany.
}

\begin{abstract}
Radon in buildings poses a significant health risk, being one of the most important causes of lung cancer deaths worldwide. Acknowledging that successful radon risk management requires engagement of stakeholders, this paper investigated prescriptions and practices for stakeholder participation. First, it points out the need to integrate radon risk management in a holistic approach to indoor air pollution, together with urban planning and energy saving policies. It then argues for establishing more systematic approaches to the involvement of stakeholders in the design, implementation and evaluation of radon actions. Finally, it suggests the development of context specific approaches for the engagement of stakeholders at local and regional level.
\end{abstract}

Keywords: indoor radon / radon action plan / stakeholder engagement / radiation protection culture / ENGAGE

\section{Introduction}

Radon in buildings poses a significant health risk, being one of the most important causes of lung cancer deaths worldwide (WHO, 2009; Gaskin et al., 2018). Information campaigns and other actions have been carried out in E.U. Member States to increase radon awareness and trigger measurement and remediation actions. While increasing awareness is an important first step to manage radon risk, it has been proven insufficient as it does not systematically lead to the application of remediation actions (WHO, 2007; Hevey, 2015). A number of studies showed that people living in areas with elevated indoor radon levels who are aware about its harmful effects are not always concerned about living in a house with high radon concentration and/or do not perform radon measurements and remediation actions (Hevey, 2015;

\footnotetext{
*Corresponding author: catrinel.turcanu@sckcen.be
}

EPA, 2019; Lofstedt, 2019). Moreover, they may not have the financial or technical resources to perform radon remediation.

Involvement of citizens from local communities and wider publics in radon risk management is deemed to help address the seemingly discrepancy between attitudes towards radon risks, and the lack of measurement and mitigation actions (Fisher and Johnson, 1990; Fisher et al., 1991; Guimond and Page, 1992; Lofstedt, 2019). Beyond local communities and citizens, a wide range of other stakeholders have been identified, that can play an important role in radon risk management, such as construction industry, building professionals, health professionals, city planners, and authorities at different levels (WHO, 2009; ICRP 2014).

Acknowledging that successful radon risk management requires engagement of stakeholders (WHO, 2009; ICRP, 2014; IAEA, 2015), this paper aimed at: i) investigating stakeholder engagement in radon risk management, as prescribed at international and national levels, and practiced at national level; and ii) highlighting opportunities for enhanced stakeholder engagement in radon risk management, 
with a view towards robust solutions to mitigating health risks from indoor radon exposures. This work has been performed within the European project ENGAGE (Turcanu et al., 2019a). In a first step, an analysis has been conducted of prescriptions for the engagement of stakeholders in radon risk management. Data were collected through i) analysis of regulatory and guidance frameworks in radon risk management, and ii) interviews $(N=4)$ with representatives of international organisations with prominent role in this field. The focus was on clarifying how are stakeholders and stakeholder engagement defined; what is the rationale for engagement; and what level of engagement is prescribed or enacted (e.g. information provision, information gathering, dialogue, collaboration, citizen actions).

Next, two types of case studies were conducted. The first set focused on engagement practices; it investigated what the issues at stake are; how the outcomes and processes of participation are crafted; what are the main challenges and opportunities; and how these practices relate to the frames set by legislative and guidance documents (Turcanu et al., 2019b). The second set of case studies focused on processes to build and transmit radiation protection (RP) culture, adapted to the different stakeholders involved in radon risk management (Barazza et al., 2019). Data for both sets of case studies were collected with document analysis and interviews. An additional cross-national case study investigated radon websites in eight countries to highlight features supporting interaction with stakeholders and informed decision-making (Perko and Turcanu, 2019).

Section 2 summarises the frameworks for stakeholder engagement in radon risk management, illustrates practices for stakeholder engagement and identifies related challenges. Based on findings, two recommendations are crystallised and detailed in section 3: i) the need for a comprehensive participatory environmental and public health protection approach to radon risk management; and ii) the benefit of developing context specific approaches for engagement of local and regional stakeholders.

\section{Frameworks and practices for stakeholder engagement in radon risk management}

\subsection{Stakeholders: a plurality of roles and responsibilities}

The Council Directive 2013/59 Euratom (2013) sets the framework for the European Member States (MS) regarding the national legislative requirements on radiological protection, including protection against radon exposures. While this document makes a specific reference to stakeholders (e.g. in the section on existing exposure situations), it does not define this term. However, it explicitly mentions some stakeholders (e.g. members of the public, local decision makers, employers and employees), and the obligation of MS to inform them about the risks associated with radon exposure. Similarly, while not defining the term "stakeholder" as such, some MS mention various stakeholders in their national legislation $(e . g$. Belgium), while others are less elaborated in this regard (e.g. Germany) (Zeleznik et al., 2019). Guidelines published by the International Atomic Energy Agency (IAEA) and World Health Organisation (WHO) identify a broad range of stakeholders such as construction industry, building professionals, researchers, radon measurement providers, authorities responsible for regulation of planning and construction of buildings (including those responsible for indoor air quality and energy efficiency), health authorities, regional and local authorities, building owners, members of the public (WHO, 2009; IAEA, 2015).

The case studies carried out in the framework of the ENGAGE project (see appendix A; and Turcanu et al., 2019b for detailed case study descriptions) recognise this broad interpretation of the concept of stakeholder. They show that radon experts (not only at national, but also at local level); organisations or companies responsible for radon remediation actions; family doctors; international organisations; school teachers; and non-governmental organisations should also be considered as stakeholders in radon risk management.

This broad range of stakeholders makes radon risk management a complex issue. First, as shown by case studies (cases 1-4, appendix A), radon risk management pertains to various fields of expertise and authority: public health, environment, geology, radiological protection, construction techniques, indoor air quality, energy savings, economic aspects, and urbanism. Bringing together these different fields is not straightforward, as they are connected to specific agendas, priorities and missions. This indicates that the management of risks related to radon exposure should be addressed in an integrated way with other policies. Examples were proposed in Belgium, by the Superior Health Council, the scientific medical authority, which recommended for radon prevention to establish a building code at all levels of legislative competences (SHC, 2017). The 2019 report of the Court of Auditors concerning the quality of private dwellings including radon proposed to address the problem of radon in an integrated way, e.g. by conditioning the granting of bonuses for energy savings in buildings located in a radon prone zone, to radon concentrations below the reference levels, or the implementation of mitigation measures to achieve this. This could prevent for instance that renovations and insulation work covered by subsidies cause increased radon levels in radon-polluted dwellings.

Second, in several countries, the governance of the aforementioned fields is situated at different levels of authority (national-regional-local), at the interplay between private and public interests. The Slovenian case study (case 2 in appendix A) highlights the lack of an appropriate organisation with involvement of all levels of public authorities to effectively perform radon control and provide remediation advice as a main challenge in the implementation of legal requirements in practice. Participants in this case study suggested that in order to make radon a priority, the government should establish a coordinating body where the radon topic would be effectively addressed. Mobilising actors at different governance levels and holding different fields of responsibility is particularly challenging in cases where there is no clear "hierarchy of norms". This is the case for instance if national authorities are in charge with radon risk management, but the responsibilities for connected domains (e.g. environment and territory management) lie under the competence of regions (Fallon, 2018, 2019). The engagement of these regional stakeholders is crucial for the success of national policies; at the same time, they should be provided with support for the implementation of those policies.

Third, actors at different governance levels may have different priorities; at provincial or local level there may be 
more attention to the specific concerns of the local population: "these [local actors] are more focused on taming and adapting to the field in their search for efficient and cheap solutions" (case study 1, appendix A, Turcanu et al., 2019b).

Finally, the need to involve the population is recognised. Radon experts participating in the Italian case study stated that "in order to move towards an improvement of the initiatives undertaken, the de facto involvement of the population is desirable, and it is on this issue that the community of radiological protection is working." (Turcanu et al., 2019a, $2019 b$ ). It is interesting to notice that the way stakeholders are defined shapes radon actions, while the scope of radon actions, in turn, shape how stakeholders are defined. For instance, focusing awareness raising campaigns only on areas with large percentages of dwellings with high radon concentrations may lead to disparities of knowledge, with particularly low awareness of radon risks outsides these zones (Fallon, 2018; confirming earlier results from Gruson et al., 2010, in Switzerland). Another example relates to radon maps. While these are useful tools for awareness raising by showing where the risk is above average, they might be misleading for local actors (Belgian case study no. 1, appendix A). Indeed, this categorisation may demobilize populations living in an area that is not referred to as a high-risk zone on the map. It also may convey the idea that radon risk is limited to a local zone, while the experience shows the contrary. This stimulates reflection on how such tools define WHO the stakeholders are, by putting certain groups in the centre of attention, and potentially leading to disengagement of those located outside these groups.

\subsection{Forms of stakeholder participation in radon risk management}

While in the past radon actions focused on awareness raising campaigns, international guidelines and regulations related to radon risk management open opportunities for enhanced stakeholder participation in radon risk management (e.g. WHO, 2009; Euratom BSS, 2013; ICRP, 2014; IAEA, 2015). The revised Basic Safety Standards Directive (2013), for instance, requires European Member States not only to develop a "Strategy for communication to increase public awareness and inform local decision makers, employers and employees of the risks of radon, including in relation to smoking" (annex XVIII), but also to "provide as appropriate for the involvement of stakeholders in decisions regarding the development and implementation of strategies for managing exposure situations" (Art. 102.). Guidelines from international organisations also emphasise, alongside dedicated radon communication and training programmes, the need for consultation of a wide range of stakeholders or interested parties when setting up reference levels (IAEA, 2015) and, more generally when establishing or implementing radon actions plans (ICRP, 2014). However, the case studies show that in most instances there is no structured approach to stakeholder engagement in the design, implementation and evaluation of national radon action plans. France provides an example of good steps in these direction, through the establishment of a national committee for the follow up of the national action plan on radon, involving representatives of different ministries (health, construction, labour, environment, $\mathrm{RP}$ authority), the experts (in RP, construction, and monitoring) and representatives of regional health agencies.

In general, two main forms of stakeholder engagement are highlighted by prescriptions and practices: 1) awareness raising on the issue of radon (a perceived prerequisite to taking action); and 2) co-developing and implementing new regulations. While the former comprises mostly information-provision and involves a broad plethora of stakeholders, including also wider publics such as homeowners and employees, the latter reflects a higher level of participation, comprising discussion and joint decision-making, and refers mostly to involvement of institutional actors. The rationales for participation also differ. In the former case, stakeholder participation is seen as an instrument to raise awareness of radon risk, increase radon measurements and the implementation of recommended mitigation actions and thus for tackling the issue of radon exposure (e.g. Euratom Basic Safety Standards, national radon action plans in several countries). In the latter case, concerning institutional stakeholders, as highlighted for instance by the WHO Handbook on indoor radon (2009), engagement is seen as key to ensuring impact, quality and sustainability of national radon programmes.

Due to lessons learned from past experiences, a general trend can be noticed, towards broadening engagement, both with respect to stakeholders (see previous subsection), as well as the level of engagement, by going beyond information provision. Examples of the latter identified in case studies include focus groups, organisation of workshops, gathering of feedback from local communities on action plans, joint inspections, providing the possibility for self-tests for radon, continuous support (including subsidies) for measurement and remediation, possibility for on-line request of a radon-detector; unaddressed post-delivery to all houses in the target municipality with support of the local authorities. Other forms of stakeholder engagement also exist that may contribute to more effective radon risk management, for example, citizen science (still rare, see for example initiatives in Ireland), commercialisation of radon tests, reducing costs or provision of tests by a non-governmental organisation. Further research should provide empirical evidence on the impact of such actions.

Radon communication should also be adapted to be more conducive to higher levels of stakeholder engagement. While social networks and internet hold potential to support engagement in radon risk management, an analysis of radon websites in eight MS (case 4, appendix A) showed that internet is insufficiently used to empower stakeholders to make informed decisions related to radon risk reduction. Some good practices that authorities could follow include, for instance, making radon information available on dedicated, easily accessible websites; providing customised information for different stakeholder groups (e.g. population, building professionals, local authority, school teachers); allowing for interaction with experts; including engaging, personalised stories; making use of social media; and publishing relevant documents related to radon action plans (Perko and Turcanu, 2019). The recent development of a cross-border web platform dedicated to radon (jurad-bat.net) is a good example of a radon website developed through cooperation of a variety of radon stakeholders from France and Switzerland. 
Table 1. Aims of developing radon radiation protection culture for different stakeholders.

\begin{tabular}{|c|c|}
\hline Stakeholder group & Aims of developing radiation protection culture \\
\hline Citizens & $\begin{array}{l}\text { Raise their awareness about radon risk in dwellings; acquire knowledge on ways to measure } \\
\text { and to remediate; increase willingness to implement measurements and remediation/protective } \\
\text { actions in their home }\end{array}$ \\
\hline $\begin{array}{l}\text { Local elected representatives and } \\
\text { administrations }\end{array}$ & $\begin{array}{l}\text { Raise awareness about radon risk in their local area; acquire knowledge on their } \\
\text { responsibilities regarding radon risk; implement measurement campaigns in their } \\
\text { municipalities (public buildings, but not only); implement or support remediation/protective } \\
\text { actions; engage action plans on radon as part of their duty to address public health issues in } \\
\text { their territories }\end{array}$ \\
\hline $\begin{array}{l}\text { Building professionals (organizations, } \\
\text { groups and workers in the field of } \\
\text { building construction and maintenance) }\end{array}$ & $\begin{array}{l}\text { Raise awareness about radon risk in buildings (dwellings, public buildings); acquire } \\
\text { knowledge on possible remediation actions; integrate radon risk at the design stage of new } \\
\text { buildings (preventive actions); integrate the radon issue in a global approach of public health } \\
\text { in buildings (in connection with indoor air quality, energy efficiency...) }\end{array}$ \\
\hline $\begin{array}{l}\text { National/local authorities (e.g. in charge } \\
\text { of RP, Health, Environment, Air } \\
\text { Quality) }\end{array}$ & $\begin{array}{l}\text { Raise their awareness about radon risk, to be involved in (support) the implementation of } \\
\text { actions such as measurement campaigns, remediation/preventive actions }\end{array}$ \\
\hline
\end{tabular}

\subsection{Development of radiation protection culture}

Case studies in France, Italy and Switzerland (no. 5 to 7 , appendix A) show that building and disseminating elements of RP culture is essential for the engagement of a wide range of stakeholders, as this encompasses not only knowledge, but also practices, attitudes, behaviours and skills related to radon risk management. Target stakeholders include, among others, inhabitants living in radon prone areas, building professionals, local/regional/national authorities responsible for the environment, urban planning, public health, energy, indoor air quality, local elected representatives, local communities, general practitioners.

The aims of developing RP culture for these stakeholders (see Tab. 1) share some commonalities (e.g. raising awareness about the health risk associated with radon exposure), but have also specificities depending on stakeholders' roles in radon risk management. Dissemination of RP culture elements relies on the use of multiple tools and processes (e.g. leaflets, training sessions, dedicated meetings). The existence of a regulatory framework for radon risk management is essential to support these processes, as it provides legitimacy to their initiators and structures upon which actions plans can be build. As radon is still unknown for a major part of the key stakeholders, development of RP culture should consider a long-term perspective, with regular evaluation and adaptation.

The case studies also illustrate that the development and implementation of actions for establishing or enhancing RP culture relies on the engagement and commitment of various stakeholders such as local and regional authorities, different ministries, universities and building professional schools.

The Italian case study (case 3 in appendix A) argues that while initiatives have been developed at regional level addressed to professionals dealing with various issues of environment and health, including training on radon together with other topics of interest, such as electromagnetic fields, air pollution, changes climate, waste, water and contaminated sites, further progress is needed in the transition from programming to a real implementation. At present, some bodies and associations in Italy (e.g. Italian National Institute of Health, Regional Environmental Protection
Agencies, regional Federations of professional engineers/architects, national order of geologists, and associations of Radiation Protection, of Qualified Experts) provide information and organise courses, but the subject of radon is not covered by basic professional training. The Belgian case study also points out that trainings are provided to architects and building professionals, among others, but the participation is very low: "The building professionals do not feel concerned and they do not believe in the interest to put a waterproof plastic cover on the soil."

\section{Towards more effective radon mitigation through enhanced stakeholder engagement}

Participants in all case studies argued that in general awareness of radon health risks is low both among the populations (particularly outside of zones classified as having highest radon risk), as well as among other key stakeholders (e.g. family doctors, architects, building professionals). Moreover, percentages of home owners in areas with high level of exposures requesting/applying remediation measures are lower than expected (Fallon, 2019).

An illustrative example is provided by a case study in Belgium, where high radon concentrations were measured in a local school. Local authorities organised public meetings, first with school teachers and parents, and subsequently with wider publics. However, there was little interest in participating to the meetings from residents WHO were not directly affected by the radon crisis in the school, and only a limited number of radon measurements in homes were carried out in the aftermath (Fallon, 2018). Experiences in France (e.g. Montbéliard and Nantes) suggest that if the local authorities are pro-active, such a situation when high radon concentrations are measured in schools could play a significant role for improving the awareness of local populations.

While there is more attention to radon in public institutions, than among private homeowners, it is not always considered a priority, both due to the low level of information or concern about the resources needed to carry out remediation (case 3, appendix A). This may lead to reluctance from 
employers to provide information about indoor radon concentrations to workers (case 2, appendix A).

The remainder of this section proposes two ways in which enhanced stakeholder engagement may lead to improved radon risk management.

\subsection{Developing comprehensive, participatory, environmental and public health protection approaches to radon risk management}

Section 2 highlighted the plurality of responsibilities (e.g. for indoor air quality, health prevention, public health protection, product regulation, management of public infrastructures), spread among authorities at different governance levels and having different objectives and approaches to risk assessment. This justifies the need for a systemic approach, connecting these different fields and authority levels. Addressing radon risks in an integrated way, together with indoor air quality and energy efficiency in the perspective of promoting the quality of buildings in a public environmental and health perspective, would enhance the effectiveness of radon risk management and, at the same time, favour the involvement of key stakeholders and the adoption of risk mitigation practices. Participation in radon risk management should be broadened to include these stakeholders in various aspects of drafting, implementation and evaluation of radon actions. This implies, among others, allowing for "open discussions about the space for negotiation, and the nature and definition of the problem" (Turnhout et al., 2010).

To ensure meaningful participation, it is essential to recognise the ethical values underlying engagement (e.g. the right to be involved of affected people) and the contribution of engagement to the quality of decision-making (e.g. in terms of co-expertise, sustainability, better solutions). Recognising such normative and substantive rationales for engagement (Stirling, 2008) and the need for joint decision-making may stimulate participants to get engaged in radon mitigation and lead to improved radon risk management. Indeed, the way engagement processes are framed, e.g. assumptions about what the issue at stake is, the expectations of the outcomes of participation, and the roles and rules of engagement have been shown to influence citizens' attitudes and response to participation (Turnhout et al., 2010).

Elaboration of actions for building and disseminating RP culture also requires participatory and multi-disciplinary approaches, involving the representatives of the target stakeholders in the elaboration of the communication media. Furthermore, involving acknowledged experts in their fields to disseminate radon knowledge and mitigation practices, rather than only radiological protection experts, has proven beneficial (e.g. involvement of Scientific and Technical Centre for Building in France, Building Engineers and Architect Schools in Switzerland).

Developing comprehensive and participatory approaches to radon risk management would first require identifying stakeholders with responsibilities for indoor air quality, public health, energy saving, construction. Second, spaces (e.g. dedicated committees) should be created allowing for interactions among the stakeholders identified, taking into account the need for a common reference frame. Furthermore, radon action plans should be integrated into public environmental and health policies (e.g. National Environmental Health Action Plans) including indoor air quality management programmes. This in turn requires establishing responsibilities for coordination of radon programmes under this comprehensive approach and providing the necessary means to carry out these responsibilities. Last but, not least, flexible communication mechanisms should be developed among the different levels of authorities.

\subsection{Strengthening the engagement of local and regional stakeholders through development of context-specific approaches}

Radon measurements and research generally take place at the national level, but mitigations occur at the local level and are very context-specific. Relevant questions such as "how would the value of houses decrease" are usually addressed at regional or provincial level.

Local initiatives, which are now being developed in many countries, are deemed very important, as national campaigns may be less effective, particularly for private houses. In Germany for instance, there are more actions at the Länder level. Länder that are more concerned with the radon problem due to a higher probability of increased radon exposure have been more actively engaged in the past by spreading information, raising awareness, bringing stakeholders together to discuss radon issues and improving education and training. More importantly, support is needed at local level to provide citizens with access to technical and financial resources for the implementation of radon risk mitigation actions.

The efficiency of local actions is reinforced when they are brought together at the regional level. The motivations are threefold. First, this would allow taking due account of the specificities of a territory. Second, it supports the sharing of experience between neighbouring municipalities confronted with similar situations. Third, it favours the mutualisation of the resources (e.g. sharing experts, organising training of building professionals, developing measurement campaigns).

Bottom-up approaches, involving local stakeholders (home owners, local construction workers, municipalities) in the elaboration and implementation of radon risk management actions, facilitate the adaptation of these actions to the local context. Indeed, engagement only at individual level may be insufficient, particularly for private home owners, as (planned and actual) behaviours (e.g. related to environment) are sensitive to the normative influence of people living in the same area (Greaves et al., 2013; Passafaro et al., 2019).

Development of context-specific local/regional approaches could first attend to adopting a systemic approach in radon action plans that considers all levels of public authorities (national, regional, local). Furthermore, stakeholders should be identified that can support at local/regional level the implementation of radon risk management actions and ensure the overall follow-up of the measurement and remediation process. These stakeholders should then be recognised by all levels of authority. Other elements supporting context-specific approaches at local/regional level include: joint assessments of the radon risk situation between local and regional actors; multi-disciplinary committees for the coordination of radon actions at the regional level; mutualisation of resources at the regional level; initiating actions at local/regional level to 
strengthen radiation protection culture; developing and testing risk communication tools (e.g. radon maps) in collaboration with local/regional stakeholders; and connecting the local/ regional campaigns for radon measurements with the campaigns for raising general public awareness.

Finally, the development and implementation of bottom-up, dynamic approaches for radon risk assessment and management at local/regional level should be supported, for instance by making radon tests available at local level; supporting citizen science; establishing networks of local radon experts; providing context-specific guidelines for risk mitigation.

\section{Discussion and conclusions}

This paper investigated prescription and practices for stakeholder engagement in radon risk management, with a view towards more robust solutions to mitigating health risks from indoor radon exposures. It also addressed the establishment of RP culture for different stakeholders and its potential role in strengthening stakeholder engagement.

Case studies showed multiple challenges connected to stakeholder engagement in radon risk management: plurality of responsibilities, lack of awareness about radon and associated risks among the general public as well as among key professional stakeholders (e.g. family doctors, architects, building professionals), disparities of knowledge (greater awareness of radon in highest radon risk areas compared to other areas), economic impacts (cost of remediation actions, as well as the potentially lower value of estate).

The paper highlighted that the framing of radon risk management actions has implications on WHO is acknowledged as a stakeholder (e.g. by authorities), or WHO recognises her/himself as a stakeholder. In order to manage effectively radon risks, it is the important to apply these actions (e.g. adaptation of building techniques) to areas larger than those having highest radon risks.

Results show that stakeholder engagement in the design, implementation and evaluation of radon risk management actions is not formalised and systematic. It is recommended to include in radon actions plans a structured approach to stakeholder engagement in the design, implementation and evaluation of actions, with recognition of the ethical and substantive rationales for stakeholder engagement. Evaluation of radon actions, jointly with the target stakeholders is a particular aspect requiring improvement.

The paper also highlighted ways that stakeholder engagement may contribute to improved radon risk management. First, it showed that the complex distribution of responsibilities and roles in radon risk management requires joint decisionmaking and integrated approaches to radon as an environmenthealth issue (related to indoor air quality) together with urban planning and energy saving policies. Participation in radon risk management should therefore be broadened to include all these stakeholders in various aspects of radon actions. In relation to RP culture for radon, the existence of a regulatory framework for radon risk management is essential to support the processes of building and disseminating RP culture elements, as it provides legitimacy to their initiators and structures to build upon action plans. Second, the importance of context specific approaches for engagement at local and regional level is brought forward, as this allows taking account the specificity of the territory, and the sharing of experiences and resources.

The argument of this paper and the case studies carried out was that stakeholder engagement is an essential part of radon risk management. Active participation of stakeholders in the design, implementation and evaluation of radon actions may improve the efficiency of radon programmes. However, even if an actor is fully empowered as a stakeholder in the radon risk management process, financial or other resources are needed to apply remediation measures. Taking into account the availability of, and fair access to, these resources for the different stakeholders has to be considered in the development of engagement processes for radon risk management.

Finally, better communication, engagement and trust building between the different radon stakeholders, with a view towards a problem-solving approach, are needed to enhance radon risk management.

Acknowledgements. The work described in this paper was conducted within the ENGAGE project, which was part of the H2020 CONCERT project. This project received funding from the EURATOM research and training programme 2014-2018 under grant agreement No. 662287.

Disclaimer (Art. 29.5 GA). This publication reflects only the author's view. Responsibility for the information and views expressed therein lies entirely with the authors. The European Commission is not responsible for any use that may be made of the information it contains.

\section{References}

Barazza F et al. 2019. Preliminary report on case studies. CONCERT Deliverable D 9.83.

EPA. 2019. National Radon Control Strategy Phase Two: 2019-2024. Report of the National Radon Control Strategy Coordination Group. Dublin: Environmental protection Agency Ireland.

Euratom BSS. 2013. COUNCIL DIRECTIVE 2013/59/EURATOM of 5 December 2013. Off. J. Eur. Union L13/1. 17.1.2014. Brussels.

Fallon C. 2018. Risk of radon and crisis of communication. In: RICOMET 2018, Antwerp, Belgium.

Fallon C. 2019. Communication and management of intervention in case of a school with high radon exposure level. In: ENGAGE final workshop, 11-13 Sep. 2019. Available from www.engage-concert.eu.

Fisher A, Johnson F. 1990. Radon risk communication research: Practical lessons. Air Waste Manage. Assoc. 40(5): 738-739.

Fisher A, McClelland GH, Schulze WD, Doyle JK. 1991. Communicating the risk from radon. Air Waste Manage. Assoc. 41(11): 1440-1445.

Gaskin J, Coyle D, Whyte J, Krewksi D. 2018. Global estimate of lung cancer mortality attributable to residential radon. Environ. Health Perspect. 126(5): 057009.

Greaves M, Zibarras LD, Stride C. 2013. Using the theory of planned behavior to explore environmental behavioral intentions in the workplace. J. Env. Psy. 34: 109-120.

Gruson M, Murith C, Rumo S. 2010. Enquête : niveau de connaissance de la population au sujet du radon en Suisse. Radioprotection 45(1): 11-30.

Guimond R, Page S. 1992. Indoor radon: A case study in risk communication. Rad. Prot. Dos. 42: 169-176.

Hevey D. 2015. Review of public information programmes to enhance home radon screening uptake and home remediation. EPA Report No 170. Ireland: Environmental Protection Agency. 
IAEA. 2015. Protection of the public against exposure indoors due to radon and other natural sources of radiation. Vienna, Austria: International Atomic Energy Agency. SSG-32.

ICRP Publication 126. 2014. Radiological Protection against Radon Exposure. Ann. ICRP 43(3).

Lofstedt R. 2019. The communication of radon risk in Sweden: Where are we and where are we going? J. Risk Res. 22(6): 773-781.

Passafaro P, Livi S, Kosic A. 2019. Local norms and the Theory of Planned Behaviour: Understanding the effects of spatial proximity on recycling intentions and self-reported ecological behaviour. Front. Psychol. 10: 744.

Perko T, Turcanu C. 2019. Is internet a missed opportunity? Evaluating radon websites from a stakeholder engagement perspective. J. Environ. Radioactiv. 212: 106123. https://doi. org/10.1016/j.jenvrad.2019.106123.

SHC. 2017. Indoor air quality in Belgium. Advisory Report of Superior Health Council No. 8794.

Stirling A. 2008. "Opening up" and "closing down" power, participation, and pluralism in the social appraisal of technology. Sci. Technol. Hum. Values 33(2): 262-294.
Turcanu C et al. 2019a. Report on venues, challenges, opportunities and recommendations for stakeholder engagement in relation to indoor radon exposure. CONCERT Deliverable 9.91.

Turcanu C et al. 2019b. Final report of the ENGAGE project. CONCERT deliverable 9.94 .

Turnhout E, Van Bommel S, Aarts MNC. 2010. How participation creates citizens: Participatory governance as performative practice. Ecol. Soc. 15(4): 26-41.

WHO. 2007. International Radon Project. Survey on Radon Guidelines, Programmes and Activities. Final report. WHO/ HSE/RAD/07.01. Geneva: World Health Organisation.

WHO. 2009. Handbook on indoor radon. A public health perspective. Geneva: World Health Organisation.

Zeleznik N et al. 2019. Rationales and frameworks for stakeholder engagement in radiation protection. CONCERT Deliverable 9.85.

\section{Appendix A Case studies on stakeholder engagement and radiological protection (RP) culture.}

Case study Research focus

1. Communication and management of intervention for a primary school with high radon exposure level (Belgium)

2. Radon action plan (Slovenia)

3. Stakeholder engagement in radon risk and prevention (Italy)

4. Stakeholder engagement through radon websites (eight EU countries)

5. Actions developed in the "Bourgogne-FrancheComté Radon pluralist project" (France)

6. Actions implemented in the framework of the Radon National Action Plan (Greece)

7. Actions implemented by in the framework of the Radon National Action Plan (Switzerland)
Dynamics of local actors (local public administration and authorities, inhabitants, teachers \& parents, medias), and interactions and communication between professional experts (local and federal levels) and other stakeholders Requirements and practices for communication and stakeholder engagement in the national radon action plan, challenges faced in the implementation of the action plan and lessons learned

Role and significance of different stakeholders' involvement in radon risk information and prevention: stakeholders actively involved, levels of involvement, exchanges of views and experience, trends in stakeholder involvement

Analysis of radon websites from national and local authorities, highlighting practices supporting stakeholder engagement: availability of radon information, accessibility, stakeholder interaction, dialogue, responsiveness, content and design, transparency and openness

Development of radiation protection culture: local actions, undertaken at the initiative of local urban public authorities of Montbéliard, in collaboration with IRSN, CEPN, Regional Agency for Air Quality Monitoring and Health Regional Agency, and with involvement of several stakeholders. These actions were developed since 2011 in a general perspective of Indoor Air Quality and energy efficiency to create awareness on radon risk and contribute to informing and supporting different actors dealing with radon management Development of radiation protection culture: actions implemented by the Greek Atomic Energy Commission in the framework of the Radon National Action Plan. Actions aimed at increasing public awareness and informing local decision makers. Involvement of ministries (Environment, Interior, Health), local authorities and building engineering organisations.

Development of radiation protection culture: actions implemented since 2012 by the Federal Office of Public Health, in collaboration with the Society of Engineers and Architects, universities of applied sciences and building professional schools, municipalities and regional authorities

Cite this article as: Turcanu C, Schieber C, Schneider T, Fallon C, Geysmans R, Perko T, Cantone M-C, Economides S, Barazza F, Charron S, Gschwind R, Lafage S, Zeleznik N, Pölzl-Viol C. 2020. Stakeholder engagement in the management of indoor radon exposures. Radioprotection 55(HS2): S227-S233 